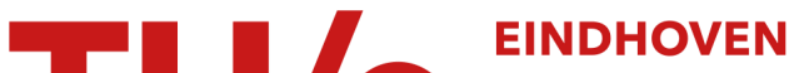 \\ UNIVERSITY OF \\ TECHNOLOGY
}

\section{Strain-vorticity induced secondary motion in shallow flows}

Citation for published version (APA):

Kamp, L. P. J. (2012). Strain-vorticity induced secondary motion in shallow flows. Physics of Fluids, 24(2), 023601-1/12. [023601]. https://doi.org/10.1063/1.3682097

DOI:

10.1063/1.3682097

Document status and date:

Published: 01/01/2012

\section{Document Version:}

Publisher's PDF, also known as Version of Record (includes final page, issue and volume numbers)

\section{Please check the document version of this publication:}

- A submitted manuscript is the version of the article upon submission and before peer-review. There can be important differences between the submitted version and the official published version of record. People interested in the research are advised to contact the author for the final version of the publication, or visit the $\mathrm{DOI}$ to the publisher's website.

- The final author version and the galley proof are versions of the publication after peer review.

- The final published version features the final layout of the paper including the volume, issue and page numbers.

Link to publication

\section{General rights}

Copyright and moral rights for the publications made accessible in the public portal are retained by the authors and/or other copyright owners and it is a condition of accessing publications that users recognise and abide by the legal requirements associated with these rights.

- Users may download and print one copy of any publication from the public portal for the purpose of private study or research.

- You may not further distribute the material or use it for any profit-making activity or commercial gain

- You may freely distribute the URL identifying the publication in the public portal.

If the publication is distributed under the terms of Article $25 \mathrm{fa}$ of the Dutch Copyright Act, indicated by the "Taverne" license above, please follow below link for the End User Agreement:

www.tue.nl/taverne

Take down policy

If you believe that this document breaches copyright please contact us at:

openaccess@tue.nl

providing details and we will investigate your claim. 


\title{
Strain-vorticity induced secondary motion in shallow flows
}

\author{
Leon P. J. Kampa) \\ Eindhoven University of Technology, Department of Applied Physics, \\ P.O. Box 513, NL-5600 MB Eindhoven, The Netherlands
}

(Received 23 December 2010; accepted 3 January 2012; published online 6 February 2012)

\begin{abstract}
Deviations from two-dimensionality of a shallow flow that is dominated by bottom friction are quantified in terms of the spatial distribution of strain and vorticity as described by the Okubo-Weiss function. This result is based on a Poisson equation for the pressure in a quasi-horizontal (primary) flow. It is shown that the Okubo-Weiss function specifies vertical pressure gradients, which for their part drive vertical (secondary) motion. An asymptotic expansion of these gradients based on the smallness of the vertical to horizontal scale ratio demonstrates that the sign and magnitude of secondary circulation inside the fluid layer is dictated by the signs and magnitude of the Okubo-Weiss function. As a consequence of this, secondary motion as well as nonzero horizontal divergence do also depend on the strength, i.e., the Reynolds number of the primary flow. The theory is exemplified by two generic vortical structures (monopolar and dipolar structures). Most importantly, the theory can be applied to more complicated turbulent shallow flows in order to assess the degree of twodimensionality using measurements of the free-surface flow only. ( 2012 American Institute of Physics. [doi:10.1063/1.3682097]
\end{abstract}

\section{INTRODUCTION}

Shallow flows might be defined as largely horizontal flows bounded by at least one boundary that is shear-supporting and a second boundary that may be stress-free or also shear-supporting. ${ }^{1}$ A well-known example is the flow inside a thin layer of fluid that is bounded by a no-slip bottom and an air-fluid interface. When the depth $H$ of this fluid layer is smaller than the typical horizontal (transverse) length scale $L$ of the flow, i.e., $\delta:=H / L \ll 1$, the fluid motion inside the layer may be qualified as being a shallow flow. For an incompressible shallow flow, a straightforward dimensional analysis of the continuity equation leads to the well-known paradigm that shallow flows in a vertically limited layer are predominantly horizontal and therefore quasi-two-dimensional (Q2D).

It is well-known that residual, i.e., vertical motion in shallow flows results from the vertical shear of the horizontal velocity field. The latter can give rise to secondary flows or pumping flows. The omnipresence of vertical motion inside shallow flows is of importance for experimental studies that deploy shallow fluid layers to investigate Q2D flows in the laboratory. For that purpose, most commonly, the fluid surface is seeded by passive tracer particles and flow measurements are performed through image analysis. These flow measurement are then confronted with numerical simulations of two-dimensional flows, where the bottom friction is modeled through a linear damping term in the two-dimensional (2D) Navier-Stokes equation and where vertical flows are ignored. However, it is well known that vertical motion inside a shallow fluid layer gives rise to a nonzero horizontal divergence of the surface flow. This compressibility leads to inhomogeneous distributions of Lagrangian tracers on such surface flows resulting in a clustering of the tracers in ridge-like structures and meandering streams. ${ }^{2-4}$

In the present paper, we will quantitatively relate magnitude and spatial distribution of vertical motion inside a shallow fluid layer to the local distribution of strain and vorticity of the surface flow.

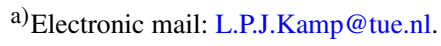


Furthermore, the compressibility of this surface flow is also quantified in terms of the local strainvorticity balance in the horizontal flow field as described by the Okubo-Weiss function. Central to our reasoning is the fact that the Okubo-Weiss function acts as a source term to the Poisson equation for the pressure.

In Sec. II, the theory that correlates vertical motions inside and nonzero horizontal divergence (compressibility) at the free surface of a shallow flow to the strain-vorticity distribution is developed. Next, this theory is applied to two elementary coherent structures found in $2 \mathrm{D}$ turbulence, a monopolar and a dipolar flow.

\section{QUASI-LINEAR THEORY OF SECONDARY MOTION IN Q2D SHALLOW FLOWS}

Vertical flows in a shallow fluid layer are assumed to be driven by vertical pressure gradients, which in turn are dictated by the vertical shear of the primary horizontal flow field. An example of such a vertical motion is the secondary flow inside a vertically confined, horizontal swirling motion (a monopolar vortex), where the cyclostrophic balance determines a horizontal pressure distribution. If this swirling flow depends upon the vertical, for example, due to a no-slip bottom, there is also a vertical pressure gradient, which drives vertical motion inside the monopolar vortex. This type of recirculation, also called Bödewadt flow, is well-known in literature. ${ }^{5}$ For more general (turbulent) shallow flows, the situation becomes more complicated and can be analyzed as follows.

Consider a Cartesian coordinate system where $x$ and $y$ denote horizontal position and the $z$-axis is along the vertical. A fluid is taken to be confined between a no-slip bottom at $z=0$ and a stress-free upper surface at $z=H$ and unbounded in the $x$-y-direction. The upper surface of this fluid layer is assumed to be flat and rigid thus excluding any free-surface deformations. For not too large flow velocities this is a justified assumption. ${ }^{6,7}$ The flow of this fluid is assumed to be predominantly horizontal and governed by the three-dimensional (3D) Navier-Stokes equation for an incompressible fluid as

$$
\frac{\partial \mathbf{v}}{\partial t}+(\mathbf{v} \cdot \nabla) \mathbf{v}=-\frac{1}{\varrho_{0}} \nabla p+v \nabla^{2} \mathbf{v},
$$

where $\varrho_{0}$ is the uniform mass density of the fluid, $p$ is the pressure, $v$ is the kinematic viscosity and $\mathbf{v}=\mathbf{v}_{\mathrm{H}}+w \mathbf{e}_{z}$, where $\mathbf{v}_{\mathrm{H}}$ is the horizontal (primary) part of the flow field, i.e.,

$$
\mathbf{v}_{\mathrm{H}}=u(x, y, z, t) \mathbf{e}_{x}+v(x, y, z, t) \mathbf{e}_{y}
$$

and $w$ is the (secondary) vertical velocity. $\mathbf{e}_{x}, \mathbf{e}_{y}$, and $\mathbf{e}_{z}$ are the unit vectors in the $x-, y$-, and $z$ direction, respectively. In what follows, we will assume that the horizontal flow field possesses a Poiseuille-like vertical structure according to

$$
\begin{aligned}
& u=\hat{u}(\mathbf{r}, t) \sin \left(\frac{\pi z}{2 H}\right), \\
& v=\hat{v}(\mathbf{r}, t) \sin \left(\frac{\pi z}{2 H}\right),
\end{aligned}
$$

where $\mathbf{r}=x \mathbf{e}_{x}+y \mathbf{e}_{y}$. Such vertical dependence of the horizontal flow field is consistent with the fact that the flow is dominated by bottom drag as will become clear later. From previous studies, ${ }^{8,9}$ it was concluded that such flows relax quickly to a Poiseuille flow, which is usually modeled by a Rayleigh bottom friction term ${ }^{10,11}$ as implied by (3) and (4) when substituted in (1).

The pressure distribution inside the fluid layer is dictated by the following Poisson equation that results from taking the divergence of (1):

$$
-\frac{1}{\varrho_{0}} \nabla^{2} p=\nabla \cdot[(\mathbf{v} \cdot \nabla) \mathbf{v}] .
$$

Consistent with results from the subsequent perturbation approach it is assumed that vertical velocities are much smaller than the horizontal ones. In that case, the right-hand side of (5) can be 
approximated by the Okubo-Weiss function $Q$, which is defined by

$$
Q=\frac{1}{2}\left(\sigma^{2}-\omega^{2}\right)
$$

with

$$
\begin{aligned}
\omega^{2} & =4 \operatorname{Det} \boldsymbol{\Omega}=\left(\frac{\partial v}{\partial x}-\frac{\partial u}{\partial y}\right)^{2}, \\
\sigma^{2} & =-4 \operatorname{Det} \mathbf{D}=\left(\frac{\partial v}{\partial x}+\frac{\partial u}{\partial y}\right)^{2}+\left(\frac{\partial u}{\partial x}-\frac{\partial v}{\partial y}\right)^{2} .
\end{aligned}
$$

Here, $\boldsymbol{\Omega}$ and $\mathbf{D}$ denote the vorticity tensor and the rate of strain tensor, respectively. Obviously $\omega$ and $\sigma$ are, respectively, the vorticity and the strain rate of the primary flow $\mathbf{v}_{\mathrm{H}}(\mathbf{r}, z, t)$.

Using the previously introduced Poiseuille-like vertical structure of the horizontal flow field $\mathbf{v}_{\mathrm{H}}$, the Poisson equation for the pressure becomes

$$
-\frac{1}{\varrho_{0}} \nabla^{2} p=\hat{Q} \sin ^{2}\left(\frac{\pi z}{2 H}\right)
$$

where

$$
\hat{Q}=\frac{1}{2}\left(\hat{\sigma}^{2}-\hat{\omega}^{2}\right)
$$

with

$$
\begin{aligned}
\hat{\omega}^{2} & =\left(\frac{\partial \hat{v}}{\partial x}-\frac{\partial \hat{u}}{\partial y}\right)^{2} \\
\hat{\sigma}^{2} & =\left(\frac{\partial \hat{v}}{\partial x}+\frac{\partial \hat{u}}{\partial y}\right)^{2}+\left(\frac{\partial \hat{u}}{\partial x}-\frac{\partial \hat{v}}{\partial y}\right)^{2}
\end{aligned}
$$

At this point, it is important to note that the Poisson equation (9) in fact relates the horizontal distribution of the magnitude of the vertical pressure gradient to the local strain-vorticity balance as quantified by the Okubo-Weiss function. This implies that also the vertical (secondary) flow and therefore the local deviation from two-dimensionality of the shallow flow is strongly correlated to the magnitude as well as the sign of $Q$. We can further develop this by solving (9). Inverting the Poisson equation (9), a particular solution for the 3D pressure distribution is given by

$$
-\frac{1}{\varrho_{0}} p(\mathbf{r}, z, t)=\int G\left(\left\|\mathbf{r}-\mathbf{r}^{\prime}\right\|, z\right) \hat{Q}\left(\mathbf{r}^{\prime}, t\right) d^{2} r^{\prime},
$$

where the Green's function for an unbounded domain is given by

$$
G(r, z)=\frac{\ln r+\cos (\gamma z) \mathrm{K}_{0}(\gamma r)}{4 \pi},
$$

with $\gamma=\pi / H$. This Green's function, being a combination of a logarithm $(\ln r)$ and a modified Bessel function $\left(\mathrm{K}_{0}(\gamma r)\right)$, is a monotonically increasing function of $r=\|\mathbf{r}\|$.

The vertical pressure gradient force that drives a vertical flow is easily found from (13):

$$
\frac{1}{\varrho_{0}} \frac{\partial p}{\partial z}=\frac{1}{4 H} \sin (\gamma z) \int \mathrm{K}_{0}\left(\gamma\left\|\mathbf{r}-\mathbf{r}^{\prime}\right\|\right) \hat{Q}\left(\mathbf{r}^{\prime}, t\right) d^{2} r^{\prime} .
$$

This relation may serve as a diagnostic equation to detect regions in the Q2D flow, where enhanced vertical motion and therefore deviations from quasi-two-dimensionality are to be expected. Relation (15) also shows that the sign of the vertical pressure gradient is determined by the sign of the Okubo-Weiss function $\hat{Q}$. Hence, the vertical motion that is driven by the vertical pressure gradient force (the left-hand side of (15)) is predominantly upward (upwelling) in rotation dominated regions $(\hat{Q}<0)$, whereas downward motion (downwelling) is concentrated in those areas where the rate of strain dominates the vorticity $(\hat{Q}>0)$. Also, upwelling fluid elements are rotating more or less without distortion but a fluid element that is pumped downward by the vertical pressure 
gradient is exponentially shrunk in one direction and exponentially elongated in other due to the local hyperbolicity of the horizontal flow. Therefore, upwelling concentrates within coherent, patchlike structures that are governed by quasi-horizontal swirling motion and areas where fluid moves downward tend to long elongated structures.

Apart from the sign of the vertical motion one may also be interested in its magnitude. Considering relation (15), it is to be expected that if $\hat{Q}<0,(>0)$, the strength of the upward (downward) pumping force is related to the magnitude of $\hat{Q}$. This can be seen as follows. Because the horizontal length scale $L$ is assumed to be much larger than the length scale $H$ on which the second part of the Green's function (that contains the modified Bessel function) varies, we expect the Okubo-Weiss function $\hat{Q}$ to be slowly varying over the region where this contribution to the Green's function in non-negligible, i.e., for $\mathbf{r}^{\prime} \approx \mathbf{r}$. Therefore, it makes sense to expand $\hat{Q}\left(\mathbf{r}^{\prime}, t\right)$ in a Taylor series around $\mathbf{r}^{\prime}=\mathbf{r}$,

$$
\begin{aligned}
\hat{Q}\left(\mathbf{r}^{\prime}, t\right)= & \hat{Q}(\mathbf{r}, t)+\nabla_{\mathrm{H}} \hat{Q}(\mathbf{r}, t) \cdot\left(\mathbf{r}^{\prime}-\mathbf{r}\right) \\
& +\frac{1}{2}\left(\mathbf{r}^{\prime}-\mathbf{r}\right)^{\mathrm{T}} \cdot \mathbf{H}(\mathbf{r}, t) \cdot\left(\mathbf{r}^{\prime}-\mathbf{r}\right)+\cdots,
\end{aligned}
$$

where $\mathbf{H}$ is the Hessian matrix of $\hat{Q}$ and $\nabla_{\mathrm{H}}$ denotes the horizontal gradient operator. When this is inserted into (13), the pressure can be expanded as follows:

$$
\begin{aligned}
p= & -\frac{\varrho_{0}}{4 \pi} \int \ln \left(\left\|\mathbf{r}-\mathbf{r}^{\prime}\right\|\right) \hat{Q}\left(\mathbf{r}^{\prime}, t\right) d^{2} r^{\prime} \\
& -\varrho_{0} \frac{H^{2}}{2 \pi^{2}} \cos (\gamma z) \sum_{n=0}^{\infty}\left(\frac{H^{2}}{\pi^{2}}\right)^{n} \triangle_{\mathrm{H}}^{n} \hat{Q}(\mathbf{r}, t),
\end{aligned}
$$

where the $n$-harmonic operator $\triangle_{\mathrm{H}}^{n}$ in cartesian coordinates is defined by

$$
\triangle_{\mathrm{H}}^{n}=\nabla_{\mathrm{H}}^{2 n}=\left(\frac{\partial^{2}}{\partial x^{2}}+\frac{\partial^{2}}{\partial y^{2}}\right)^{n} .
$$

Rescaling the horizontal coordinates $x$ and $y$ with $L$, the vertical coordinate $z$ with $H$, time $t$ with $H^{2} / \nu$, all velocity components with some characteristic velocity $U$, pressure $p$ with $\varrho_{0} U^{2}$, and finally the Okubo-Weiss function with $U^{2} / L^{2}$, rewrites (17) into the following dimensionless form:

$$
\begin{aligned}
p & =-\frac{1}{4 \pi} \int \ln \left(\left\|\mathbf{r}-\mathbf{r}^{\prime}\right\|\right) \hat{Q}\left(\mathbf{r}^{\prime}, t\right) d^{2} r^{\prime} \\
& -\frac{\delta^{2}}{2 \pi^{2}}\left[\hat{Q}(\mathbf{r}, t)+\frac{\delta^{2}}{\pi^{2}} \Delta_{\mathrm{H}} \hat{Q}(\mathbf{r}, t)+\cdots\right] \cos (\pi z),
\end{aligned}
$$

which shows that (17) represents a series expansion for the pressure in terms of the small parameter $\delta^{2}=H^{2} / L^{2}$. It turns out that to lowest order the pressure is determined by the Okubo-Weiss function itself; whereas, in the next order of this expansion contributions to the pressure are determined by the mean curvature $\left(=\operatorname{trace}(\mathbf{H})=\nabla^{2} \hat{Q}\right)$ of the $2 \mathrm{D}$ surface $z=\hat{Q}(x, y, t)$. From expansion (19) the pressure gradients are found to be given by

$$
\begin{aligned}
\nabla p= & -\frac{1}{4 \pi} \int \frac{\mathbf{r}-\mathbf{r}^{\prime}}{\left\|\mathbf{r}-\mathbf{r}^{\prime}\right\|^{2}} \hat{Q}\left(\mathbf{r}^{\prime}, t\right) d^{2} r^{\prime} \\
& -\frac{\delta^{2}}{2 \pi^{2}} \nabla[\hat{Q}(\mathbf{r}, t) \cos (\pi z)]+O\left(\delta^{4}\right) .
\end{aligned}
$$

This result shows that to lowest-order the horizontal pressure gradient is independent of the vertical coordinate $z$ and may be interpreted as the depth-averaged cyclostrophic balance. The shallow flow expansion of the vertical pressure gradient demonstrates that its local strength is to lowest order determined by the sign and magnitude of the Okubo-Weiss function. Therefore, it is to be expected that the strongest vertical motion that is driven by this vertical pressure gradient is to be found within structures of high strain or high vortical activity. Although it is clear that identifying these structures in a given flow field involves the critical points of the Okubo-Weiss function, their extraction is not 
trivial but might be feasible using the recently introduced vortex and strain skeletons ${ }^{12}$ or curvature fields constructed from passive tracer trajectories. ${ }^{13}$

Once the vertical pressure gradient is known from (20), the (dimensionless) vertical fluid velocity $w$ that is driven by this force can be found from the following (dimensionless) $z$-component of the 3D Navier-Stokes equation:

$$
\frac{\partial w}{\partial t}+\delta \operatorname{Re}\left[\delta\left(\mathbf{v}_{\mathrm{H}} \cdot \nabla_{\mathrm{H}} w\right)+w \frac{\partial w}{\partial z}\right]-\left(\delta^{2} \triangle_{\mathrm{H}}^{1} w+\frac{\partial^{2} w}{\partial z^{2}}\right)=-\delta \operatorname{Re} \frac{\partial p}{\partial z}=-\delta^{3} \operatorname{Re} \frac{\hat{Q}(\mathbf{r}, t)}{2 \pi} \sin (\pi z)+O\left(\delta^{5}\right),
$$

where $\operatorname{Re}=U L / v$ is the Reynolds number pertinent to the horizontal flow. For

$$
1 \ll \sqrt{\operatorname{Re}} \ll 1 / \delta
$$

to lowest order the vertical velocity is governed by

$$
\left(\frac{\partial}{\partial t}-\frac{\partial^{2}}{\partial z^{2}}\right) w=-\delta^{3} \operatorname{Re} \frac{\hat{Q}(\mathbf{r}, t)}{2 \pi} \sin (\pi z)+O\left(\delta^{5}\right) .
$$

From the continuity condition, it is then found that the horizontal divergence to this order in the approximation is determined by

$$
\left(\frac{\partial}{\partial t}-\frac{\partial^{2}}{\partial z^{2}}\right)\left(\nabla_{\mathrm{H}} \cdot \mathbf{v}_{\mathrm{H}}\right)=\delta^{2} \operatorname{Re} \frac{\hat{Q}(\mathbf{r}, t)}{2} \cos (\pi z)+O\left(\delta^{4}\right) .
$$

The vertical velocity $w$ and the horizontal divergence $\nabla_{\mathrm{H}} \cdot \mathbf{v}_{\mathrm{H}}$ both signify departure from presumed two-dimensionality of the shallow flow that is considered here. Both Eqs. (23) and (24) clearly demonstrate that the degree of two-dimensionality of the primary quasi-horizontal flow not only depends on the shallowness $(\delta$ ) of the fluid layer but also on the magnitude of this primary flow (Re). The scaling of $w$ and $\nabla_{\mathrm{H}} \cdot \mathbf{v}_{\mathrm{H}}$ with $\delta$ and $\mathrm{Re}, \delta^{3} \mathrm{Re}$, and $\delta^{2} \mathrm{Re}$, respectively, is consistent with recent findings by Duran-Matute et al. ${ }^{8,14}$ for decaying monopolar and dipolar vortices. Moreover, Eqs. (23) and (24) relate the three-dimensionality of the shallow flow to the local magnitude of the Okubo-Weiss function $\hat{Q}(\mathbf{r}, t)$. The right-hand side of Eq. (24) not being zero is implicated by the compressibility of the free-surface flow $\left(\nabla_{\mathrm{H}} \cdot \mathbf{v}_{\mathrm{H}}=-\partial w / \partial z \neq 0\right)$. Overall mass conservation dictates that regions on the upper surface where $\nabla_{\mathrm{H}} \cdot \mathbf{v}_{\mathrm{H}}>0(<0)$ imply upwelling (downwelling) of the fluid below. More specifically, it is seen that upwelling (downwelling) and positive (negative) horizontal divergence is concentrated in vorticity (strain) dominated regions of the primary flow. In fact, when a steady-state is attained, the horizontal divergence at the upper free surface of the shallow fluid layer as well as the vertical velocity at half-depth can both be considered as footprints of the local Okubo-Weiss function that is associated with the quasi-horizontal flow.

It should be clear that a nonzero horizontal divergence will result in stretching of vortex lines in the $z$-direction. This will eventually modify the vertical vorticity $\omega$ of the primary flow. This feedback of the secondary flow on the primary motion is not taken into account in the present analysis. Equation (24) shows that this feedback scales with $\delta^{2}$ Re provided inequalities (22) are satisfied. In fact, these inequalities express that not only the flow is shallow but also that the thickness of the boundary layer above the no-slip bottom is comparable or even larger than the depth $H$ of the fluid layer. Hence, the shallow flow is dominated by bottom friction and as has been shown by Duran-Matute $e t a l{ }^{8}$ for flows in the regime determined by (22), the vertical structure quickly relaxes to Poiseuille-like profile. This a posteriori justifies the presumed Poiseuille-like vertical structure of the horizontal flow (see (3) and Ref. 9).

It is well-known that the inverse energy cascade in 2D turbulence leads to large-scale coherent structures. Monopolar and dipolar vortices seem to be the generic building blocks of these flow structures, which are often studied experimentally in shallow fluid layers (in order to promote the two-dimensionality of the flow). Therefore, it makes sense to apply the results found in this section to these flow structures in order to assess the role of vertical motion in Q2D turbulence. 


\section{VERTICAL MOTION INSIDE AN AXISYMMETRIC MONOPOLAR VORTEX}

Consider a circularly symmetric, swirling flow that in terms of cylindrical coordinates $r, \theta$, and $z$ is given by

$$
\mathbf{v}=\left(v_{r}, v_{\theta}, v_{z}\right)=\hat{v}_{\theta}(r) \sin \left(\frac{\pi z}{2 H}\right) \mathbf{e}_{\theta},
$$

where $\mathbf{e}_{\theta}$ is the unit vector in the $\theta$-direction and we have assumed a Poiseuille-like vertical structure. Introducing a characteristic magnitude $U$ for the azimuthal velocity, $\hat{v}_{\theta}(r)$ is written as

$$
\hat{v}_{\theta}(r)=U \mathrm{f}(\rho),
$$

where

$$
\rho=\frac{r}{R},
$$

with $R$ a the radius of the monopolar vortex. The function $\mathrm{f}(\rho)$ models the radial dependence of the swirl velocity and its magnitude is assumed to be of order unity. Therefore, the Reynolds number becomes $\operatorname{Re}=U R / v$, where $v$ is the kinematic viscosity. Typical examples of $\mathrm{f}(\rho)$ that model experimentally observed barotropic monopolar vortices are

$$
\mathrm{f}(\rho)=\frac{1}{2 \rho}\left(1-e^{-\rho^{2}}\right),
$$

which is the Lamb-Oseen vortex and

$$
\mathrm{f}(\rho)=\frac{\rho}{2} e^{-\rho^{2}}
$$

which is the Gaussian (shielded) vortex.

The vorticity and strain rate as introduced in Sec. II attain a particularly simple form for these circularly symmetric, monopolar vortices,

$$
\omega=\frac{1}{r} \frac{\partial}{\partial r}\left(v_{\theta}\right), \quad \sigma=r \frac{\partial}{\partial r}\left(\frac{v_{\theta}}{r}\right) .
$$

From these, the Okubo-Weiss function is found to be given by

$$
Q=-\frac{1}{r} \frac{\partial}{\partial r}\left(v_{\theta}^{2}\right)=\hat{Q}(\rho) \sin ^{2}\left(\frac{\pi z}{2 H}\right),
$$

where

$$
\hat{Q}(r)=-\frac{1}{r} \frac{d}{d r}\left[\hat{v}_{\theta}^{2}(r)\right]=\hat{Q}_{0} \frac{1}{\rho} \frac{d}{d \rho}\left[\mathrm{f}^{2}(\rho)\right]
$$

where

$$
\hat{Q}_{0}=-\frac{U^{2}}{R^{2}} .
$$

Introducing the aspect ratio $\delta=H / R$ and the new variables $\eta=\rho / \delta=r / H$ and $\zeta=z / H$, the Poisson equation (9) becomes

$$
\frac{\partial^{2} p}{\partial \eta^{2}}+\frac{1}{\eta} \frac{\partial p}{\partial \eta}+\frac{\partial^{2} p}{\partial \zeta^{2}}=\frac{1}{2 \eta} \frac{d}{d \eta}\left[\mathrm{f}^{2}(\delta \eta)\right][1-\cos (\pi \zeta)],
$$

where the pressure has been replaced by $\varrho_{0} U^{2} p$. Equation (32) can be solved using the same Green's function formalism that has been used in Sec. II. Thus, the dimensionless pressure $p$ is found to be given by

$$
p(\eta, \zeta)=\int_{0}^{\eta} \frac{\mathrm{f}^{2}\left(\delta \eta^{\prime}\right)}{2 \eta^{\prime}} d \eta^{\prime}+\frac{\pi}{2} \cos (\pi \zeta) \int_{0}^{\infty} G\left(\eta, \eta^{\prime}\right) \mathrm{f}^{2}\left(\delta \eta^{\prime}\right) d \eta^{\prime}+p_{0},
$$

where $p_{0}$ is an arbitrary constant and

$$
G\left(\eta, \eta^{\prime}\right)=\left\{\begin{array}{l}
+\mathrm{I}_{0}(\pi \eta) \mathrm{K}_{1}\left(\pi \eta^{\prime}\right) \text { if } \eta<\eta^{\prime}<\infty, \\
-\mathrm{K}_{0}(\pi \eta) \mathrm{I}_{1}\left(\pi \eta^{\prime}\right) \text { if } 0<\eta^{\prime}<\eta,
\end{array}\right.
$$


with $\mathrm{I}_{0}$ and $\mathrm{I}_{1}$ modified Bessel functions of the first kind of zeroth and first order, respectively, and $\mathrm{K}_{0}$ and $\mathrm{K}_{1}$ modified Bessel functions of the second kind. From this result, the two pressure gradients are found to be

$$
\begin{aligned}
\frac{\partial p}{\partial \eta}= & \frac{\mathrm{f}^{2}(\delta \eta)}{2 \eta}[1-\cos (\pi \zeta)]+\frac{\pi^{2}}{2} \cos (\pi \zeta) \\
& \cdot\left[\mathrm{K}_{1}(\pi \eta) \int_{0}^{\eta} \mathrm{I}_{1}\left(\pi \eta^{\prime}\right) \mathrm{f}^{2}\left(\delta \eta^{\prime}\right) d \eta^{\prime}+\mathrm{I}_{1}(\pi \eta) \int_{\eta}^{\infty} \mathrm{K}_{1}\left(\pi \eta^{\prime}\right) \mathrm{f}^{2}\left(\delta \eta^{\prime}\right) d \eta^{\prime}\right], \\
\frac{\partial p}{\partial \zeta}= & \frac{\pi^{2}}{2} \sin (\pi \zeta) \cdot\left[\mathrm{K}_{0}(\pi \eta) \int_{0}^{\eta} \mathrm{I}_{1}\left(\pi \eta^{\prime}\right) \mathrm{f}^{2}\left(\delta \eta^{\prime}\right) d \eta^{\prime}-\mathrm{I}_{0}(\pi \eta) \int_{\eta}^{\infty} \mathrm{K}_{1}\left(\pi \eta^{\prime}\right) \mathrm{f}^{2}\left(\delta \eta^{\prime}\right) d \eta^{\prime}\right] .
\end{aligned}
$$

The first of these two equations expresses the so-called cyclostrophic balance that is modified by the Poiseuille-like $z$-dependence of the swirling motion. The second equation denotes the vertical pressure gradient that results from the $z$-dependence of the horizontal swirl velocity. Straightforward asymptotic analysis of these pressure gradients for $\delta \downarrow 0$ leads to

$$
\begin{aligned}
& \frac{\partial p}{\partial \eta}=\delta \frac{\mathrm{f}^{2}(\rho)}{2 \rho}+O\left(\delta^{2}\right), \\
& \frac{\partial p}{\partial \zeta}=-\delta^{2} \frac{1}{2 \pi \rho} \frac{d}{d \rho}\left[\mathrm{f}^{2}(\rho)\right] \sin (\pi \zeta)+O\left(\delta^{3}\right) .
\end{aligned}
$$

Expressed in dimensionfull quantities these become in lowest order

$$
\begin{aligned}
\frac{1}{\varrho_{0}} \frac{\partial p}{\partial r} & =\frac{\hat{v}_{\theta}^{2}(r)}{2 r}, \\
\frac{1}{\varrho_{0}} \frac{\partial p}{\partial z} & =-\frac{H}{2 \pi r} \frac{d}{d r}\left[\hat{v}_{\theta}^{2}(r)\right] \sin \left(\frac{\pi z}{H}\right) \\
& =\frac{H}{2 \pi} \hat{Q}(r) \sin \left(\frac{\pi z}{H}\right) .
\end{aligned}
$$

Note that the lowest-order contribution to the radial pressure gradient as given by (41) does not depend on the vertical coordinate $z$ and therefore differs from what is expected from the conventional cyclostrophic balance, i.e.,

$$
\frac{1}{\varrho_{0}} \frac{\partial p}{\partial r}=\frac{v_{\theta}^{2}(r)}{r}=\frac{\hat{v}_{\theta}^{2}(r)}{r} \sin ^{2}\left(\frac{\pi z}{2 H}\right) .
$$

In fact, (41) may be considered as the depth-averaged cyclostrophic balance.

The vertical motion that is driven by the vertical pressure gradient (40) is governed by the $z$-component of the Navier-Stokes equation, i.e.,

$$
\frac{\partial w}{\partial t}+\operatorname{Re} \delta\left(\delta u \frac{\partial w}{\partial \rho}+w \frac{\partial w}{\partial \zeta}\right)-\left[\delta^{2} \frac{1}{\rho} \frac{\partial}{\partial \rho}\left(\rho \frac{\partial w}{\partial \rho}\right)+\frac{\partial^{2} w}{\partial \zeta^{2}}\right]=-\delta \operatorname{Re} \frac{\partial p}{\partial \zeta},
$$

where $u=v_{r} / U$ and $w=v_{z} / U$. For $1 \ll \sqrt{\operatorname{Re}} \ll 1 / \delta$, the lowest order steady-state solution of this equation is given by

$$
w=\delta^{3} \operatorname{Re} \frac{1}{2 \pi^{2} \rho} \frac{d}{d \rho}\left[\mathrm{f}^{2}(\rho)\right] \sin (\pi \zeta) .
$$

Moreover, from the incompressibility condition the radial velocity $u$ is found to be given by

$$
u=-\delta^{2} \operatorname{Re} \frac{1}{2 \pi \rho} \mathrm{f}^{2}(\rho) \cos (\pi \zeta)
$$

to the present order in the approximation. 
Equations (45) and (46) clearly demonstrate that associated with the primary swirl flow $v_{\theta}$ a secondary, recirculating flow $v_{r}$ and $v_{z}$ is set up with upwelling in the core of the monopolar vortex (where vorticity dominates, i.e., $Q<0$ ), radial outflow near the upper surface of the fluid layer, downwelling at the outskirts of the vortex (where strain dominates, i.e., $Q>0$ ) and radial inflow close to the bottom. This type of recirculation that takes place in vortices that have their rotation axes normal to a solid bottom has already been described by Bödewadt in 1940. ${ }^{5,15}$ In fact, as early as in 1926 Einstein presented the solution of the tea leaves paradox (why do tea leaves at the bottom of a tea cup migrate to the center of the cup after the water is stirred?) in terms of such recirculating flows (see Ref. 16).

\section{VERTICAL MOTION INSIDE A LAMB-CHAPLYGIN DIPOLE}

The Lamb-Chaplygin vortex dipole is considered to be a realistic representation of horizontal slices of experimentally created dipolar vortices. ${ }^{17-21}$ In a frame co-moving with the dipole, the horizontal flow in a Lamb-Chaplygin dipole is described by the following stream function given in cylindrical coordinates $(r, \theta):^{22}$

$$
\hat{\psi}(x, y)= \begin{cases}-2 \frac{U}{k} \frac{\mathrm{J}_{1}(k r)}{\mathrm{J}_{0}(k L)} \sin \theta & \text { if } 0<r<L, \\ U\left(\frac{L^{2}}{r^{2}}-1\right) r \sin \theta & \text { if } r>L,\end{cases}
$$

where $r^{2}=x^{2}+y^{2}$ and $\tan \theta=y / x$. $\mathbf{J}_{n}$ is the $n$th order Bessel function of the first kind and $L$ is the dipole's horizontal length scale. $U$ now is the (steady) translation speed of the dipole and the constant $k$ is determined by $\mathbf{J}_{1}(k L)=0$, which corresponds with the first zero $j_{1,1}$ of $\mathbf{J}_{1}$.

The Okubo-Weiss function is a Eulerian quantity that is Galilean invariant and therefore can be evaluated in the frame co-moving with the dipole. $\hat{Q}$ is readily obtained from stream function (47) using that

$$
\hat{Q}=2\left(\hat{\psi}_{x y}^{2}-\hat{\psi}_{x x} \hat{\psi}_{y y}\right)
$$

Outside the dipole, i.e., for $x^{2}+y^{2}>L^{2}, \hat{Q}$ is found to be given by

$$
\hat{Q}_{e}=\hat{Q}_{0}\left(\frac{\rho_{0}}{\rho}\right)^{6} \text {. }
$$

For the dipolar flow with $x^{2}+y^{2}<L^{2}$, we obtain

$$
\hat{Q}_{i}=\hat{Q}_{0} \frac{\mathbf{J}_{2}(\rho)\left[\mathbf{J}_{2}(\rho)-\rho \mathbf{J}_{1}(\rho) \sin ^{2} \theta\right]}{\mathbf{J}_{0}^{2}\left(\rho_{0}\right)}\left(\frac{\rho_{0}}{\rho}\right)^{2},
$$

where

$$
\begin{aligned}
& \rho=k r=k \sqrt{x^{2}+y^{2}}, \rho_{0}=k L=j_{1,1} \simeq 3.83170597, \\
& \text { and } \hat{Q}_{0}=8\left(\frac{U}{L}\right)^{2} .
\end{aligned}
$$

Outside the separatrix $\rho=\rho_{0}$, the flow is potential and therefore in this outer region the OkuboWeiss function is positive definite. Inside the separatrix, $\hat{Q}$ attains positive as well as negative values. Figure 1 shows a surface plot of $\hat{Q}$ in the frame co-moving with the dipole and normalized by its (constant) value on the separatrix. The white contour is defined by $\hat{Q}=0$ and separates the vortical regions from the strain-dominated domains. Outside the separatrix $\hat{Q}$ is a monotonically decreasing function for increasing $r$. However, inside the dipolar flow region the Okubo-Weiss function has extremal features that consist of two (one-dimensional) absolute minima that are close to the location of the two vortex cores and two (one-dimensional) absolute maxima on the $x$-axis. The latter are close to the location of the the two hyperbolic (stagnation) points of the flow field, but shifted somewhat inwards towards the origin, see Fig. 1. Also, $\hat{Q}$ attains a (two-dimensional) relative 


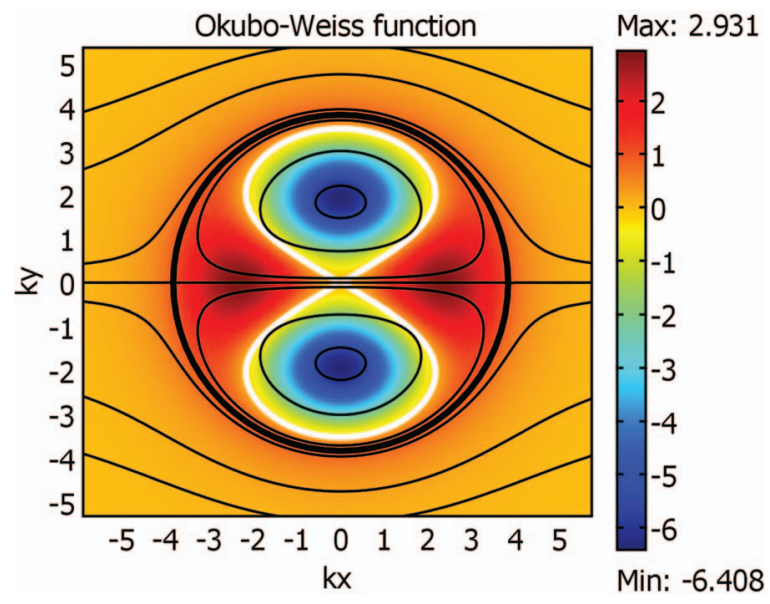

FIG. 1. (Color online) Surface plot of the normalized Okubo-Weiss function $\hat{Q}$ for a Lamb-Chaplygin dipole. The white contour separates the region where $\hat{Q}>0$ (outside the closed curve) from that where $\hat{Q}>0$ (inside the contour). The boldface black circle is the separatrix. The other black contours denote streamlines of the dipolar flow in the frame co-moving with the dipole.

maximum in the form of a height ridge at the location of the separatrix. Finally, we note that $\hat{Q}$ also possesses a critical point of saddle type at the origin. Furthermore, we observe that $\hat{Q}$ is continuous everywhere but its derivatives are discontinuous at the separatrix. Therefore, expansions (16) and (17) are not valid around $\rho=\rho_{0}$, and we need to solve for the vertical pressure gradient using (15) instead.

Figure 2 shows a surface plot of the nondimensionalized vertical pressure gradient at half depth and evaluated from the right-hand side of expression (15) with $\delta=0.7$. The white contour indicates where this pressure gradient becomes zero. Obviously, inside the two vortex cores the vertical pressure gradient tends to drive fluid upward. Outside the white contour and most powerful close to the two stagnation points, slightly shifted inward towards the origin, fluids tends to be driven downward.

An important difference between the present example and the monopolar vortex of the previous section is that in the present dipolar flow downwelling of fluid takes place close to hyperbolic stagnation points. The latter implies that due to higher-order (convective) effects the areas where this downwelling takes place will be exponentially shrunk (elongated) in the direction of the stable

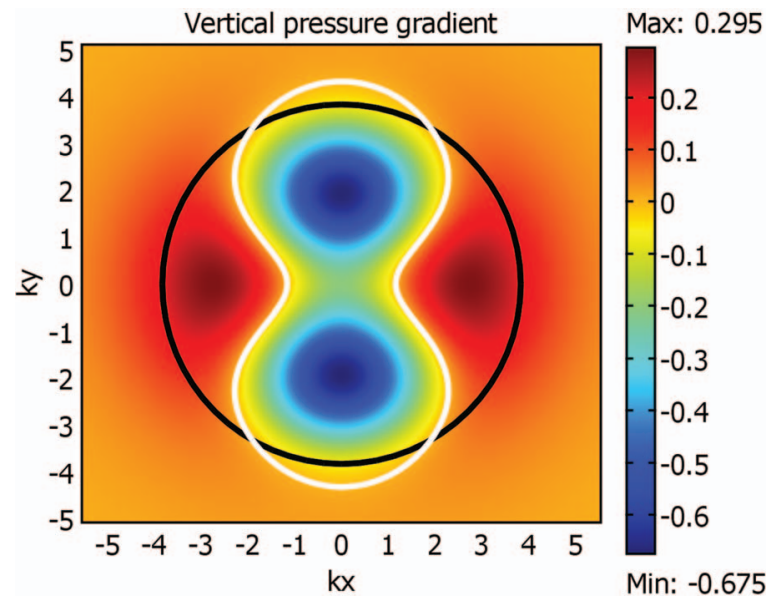

FIG. 2. (Color online) Surface plot of the nondimensionalized vertical pressure gradient as given by (15) for $z=H / 2$. The white contour separates the regions of positive and negative gradients. The boldface black circle is the separatrix. 
(a) Vertical velocity

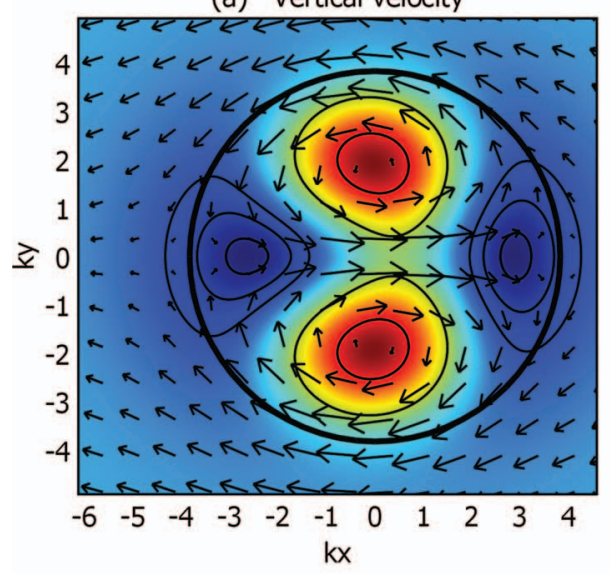

(b) Vertical velocity

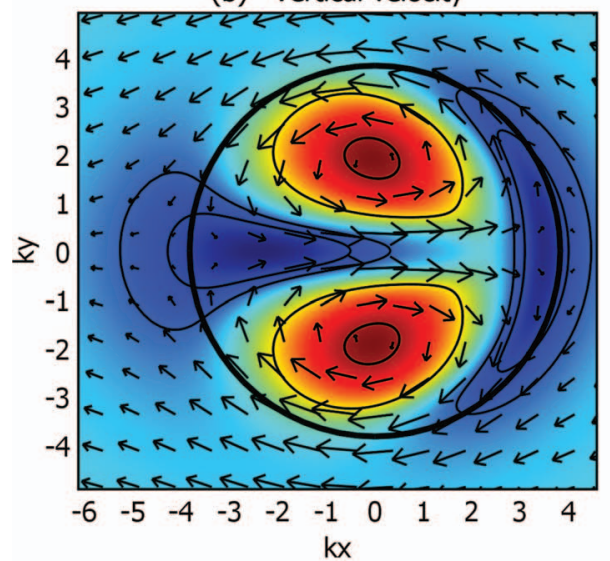

(c) Vertical velocity

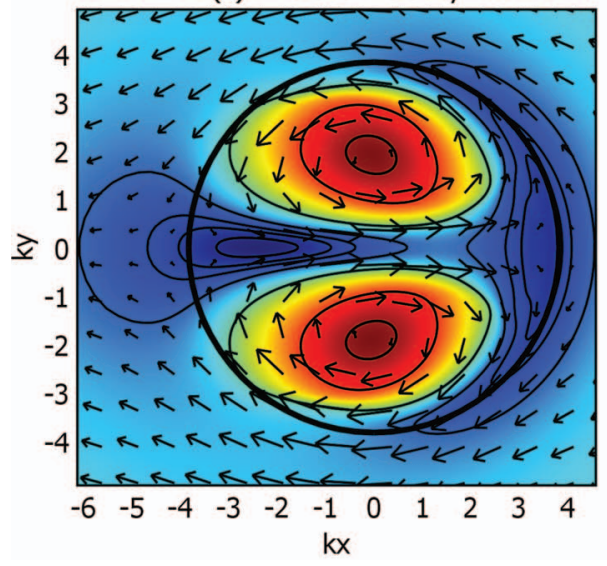

Max: 0.335

0.3

$-0.25$

$-0.2$

$-0.15$

$-0.1$

0.05

$-0$

$-0.05$

$-0.1$

Min: -0.147

Max: 1.077

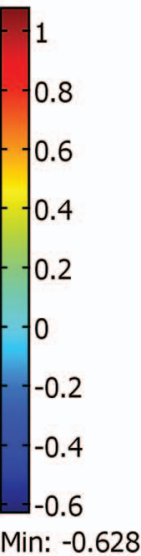

Max: 1.093

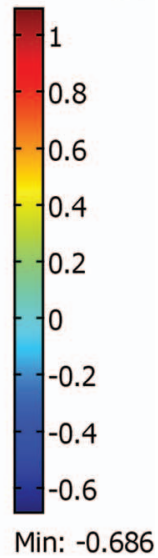

FIG. 3. (Color online) Numerically obtained snapshots of the vertical velocity as determined by the solution of (21) with $\delta$ $=0.7$ and $\operatorname{Re}=1000$ at $t=0.5 / \mathrm{kU}(\mathrm{a}), t=3 / \mathrm{kU}(\mathrm{b})$, and $t=5 / \mathrm{kU}(\mathrm{c})$. The arrows denote the the horizontal flow field and the black contours are iso-lines of the vertical velocity. The boldface black circle is the separatrix.

(unstable) manifold that is associated with these points. This effect is illustrated in Fig. 3, which shows the temporal evolution of the vertical velocity as obtained from the numerical solution of the full convection-diffusion equation (21) with $\delta=0.7$ and $\mathrm{Re}=U L / v=1000$. These snapshot are taken in the frame co-moving with the dipole and the (steady) horizontal flow is derived from the nondimensionalized stream function (47) according to $\mathbf{v}_{\mathrm{H}}=\nabla_{\mathrm{H}} \times\left(\hat{\psi} \mathbf{e}_{z}\right)$. Obviously, as time 
advances, the magnitude of the vertical velocity increases and also the areas where downwelling and upwelling takes place do change in time. Especially, the frontal downwelling can be seen to gradually deform into a thin elongated structure that tends to delineate the instantaneous separatrix that is associated with the frontal stagnation point. The downwelling in the back of the dipole is elongated along the dipole's symmetry axis. This evolution is confirmed by recently obtained experimental results on the dynamics and structure of decaying shallow dipolar vortices. ${ }^{14}$

\section{CONCLUSIONS}

Vertical or secondary motion in a shallow, quasi-two-dimensional flow is driven by vertical pressure gradients, which are inherited from vertical gradients in the primary quasi-horizontal flow. These gradients are inevitable for a flow in a single fluid layer above a no-slip bottom and can also be present in more elaborate setups that try to suppress these gradients like two-fluid-layer setups. Using a formal perturbation approach and the relation between the Okubo-Weiss function and the pressure, the latter is expanded in terms of the aspect ratio $\delta$. Thus, it is shown that upwelling and downwelling of fluid in a shallow flow is to lowest order, i.e., for flows that are dominated by bottom friction, determined by the sign and local magnitude of the Okubo-Weiss function. Therefore and consistent with recent findings for decaying monopolar and dipolar vortices, the strength of this vertical motion scales not only with the aspect ratio but also with the Reynolds number Re that can be associated with quasi-horizontal shallow flow. More specifically, it has been shown that the vertical motion scales with $\delta^{3} \operatorname{Re}$ and the horizontal divergence with $\delta^{2} \mathrm{Re}$. These scalings also signify that departure from two-dimensionality in shallow flows needs to be judged from the aspect ratio as wel as from the flow's Reynolds number.

It has been demonstrated that the higher-order advection terms in the convection-diffusion, Eq. (21) will result in horizontal advection of the up- and downwelling areas. Since the downwelling takes place predominantly in strain-dominated regions, the local hyperbolicity of the flow will reshape these downwelling areas into long elongated structures. On the other hand, upwelling is concentrated in vorticity-dominated areas that are not much distorted by the horizontal advection. This behavior has important consequences for the dynamics of passive Lagrangian tracers that are advected in such flows. ${ }^{2}$

The retroaction of the secondary motion on the primary flow is not modeled here. However, it is clear that such a feedback will result in a modification of the primary flow mainly due to vortex stretching and tilting as a result of the non-vanishing horizontal divergence. Up-flow and related outflow at the top of a vortex core will reduce the vertical vorticity close to the upper surface of the fluid layer. This is basically due to conservation of angular momentum: the swirl velocity of the radially spreading fluid decreases. Similarly, close to the base of a vortex core vertical vorticity tends to be enhanced by the related inflow of fluid. Thus, vertical vorticity is redistributed, which implies alterations of the horizontal velocity as well as the Okubo-Weiss function. Also, the temporal decay of the primary flow due to the bottom friction has not been considered here. Since we assume a Poiseuille-like vertical profile for the horizontal velocities, this decay can be modeled by Rayleigh friction. The nondimensionalized Rayleigh decay rate is given by $\pi^{2} / 4$ and the nondimensionalized rate of advection by the horizontal flow is is given by $\delta^{2} \mathrm{Re}$. Therefore, the strain induced elongation of downwelling areas in decaying hyperbolic flow as exampled for a dipolar flow (see Fig. 3) will only evolve if $\delta^{2} \operatorname{Re} \geq \pi^{2} / 4$. The latter and the quantification of vertical velocities as well as horizontal divergence (compressibility) as described in this paper are of relevance to Eulerian and Lagrangian studies in surface flow dynamics in shallow fluid layers.

Finally, it should be noted that the results presented in this paper are based on several assumptions. First of all the vertical structure of the quasi-horizontal flow is assumed to be Poiseuille-like. Previous studies have shown that for shallow flows that are dominated by bottom drag, as is the case in the present approach, this is a legitimate presumption. Secondly, deformations of the free surface of the fluid layer have been ignored. Any vertical motion associated with such deformations is therefore excluded. For Reynolds numbers within the regime considered in this paper, depth variations 
in the fluid layer due to surface gravity waves or localized depressions due to swirling motion are generally weak as has been concluded in other studies.

${ }^{1}$ G. H. Jirka and W. S. J. Uijttewaal, Shallow Flows (Balkema, Leiden, 2004), p. 3.

${ }^{2}$ J. Schumacher and B. Eckhardt, "Clustering dynamics of Lagrangian tracers in free-surface flows," Phys. Rev. E 66, 017303 (2002).

${ }^{3}$ J. R. Cressman, J. Davoudi, W. I. Goldburg, and J. Schumacher, "Eulerian and Lagrangian studies in surface flow turbulence," New J. Phys. 6, 53 (2004).

${ }^{4}$ A. R. Cieslik, L. P. J. Kamp, H. J. H. Clercx, and G. J. F. van Heijst, "Meandering streams in a shallow fluid layer," Europhys. Lett. 85, 54001 (2008).

${ }^{5}$ H. P. Greenspan, The Theory of Rotating Fluids (Cambridge University Press, London, 1969).

${ }^{6}$ R. A. D. Akkermans, L. P. J. Kamp, H. J. H. Clercx, and G. J. F. van Heijst, "Intrinsic three-dimensionality in electromagnetically driven shallow flows," Europhys. Lett. 83, 24001 (2008).

${ }^{7}$ R. A. D. Akkermans, A. R. Cieslik, L. P. J. Kamp, H. J. H. Clercx, and G. J. F. van Heijst, "The three-dimensional structure of an electromagnetically generated dipolar vortex in a shallow fluid layer," Phys. Fluids 20, 116601 (2008).

${ }^{8}$ M. Duran-Matute, L. P. J. Kamp, R. R. Trieling, and G. J. F. van Heijst, "Scaling of decaying shallow axisymmetric swirl flows," J. Fluid Mech. 648, 509 (2010).

${ }^{9}$ M. P. Satijn, A. W. Cense, R. Verzicco, H. J. H. Clercx, and G. J. F. van Heijst, “Three-dimensional structure and decay properties of vortices in shallow fluid layers," Phys. Fluids 13, 1932 (2001).

${ }^{10}$ F. V. Dolzhanski, V. A. Krymov, and D. Yu. Manin, "An advanced experimental investigation of quasi-two-dimensional shear flows," J. Fluid Mech. 241, 705 (1992).

${ }^{11}$ G. Boffetta, A. Cenedese, S. Espa, and S. Musacchio, "Effects of friction on 2D turbulence: An experimental study of the direct cascade," Europhys. Lett. 71, 590 (2005).

${ }^{12}$ J. Sahner, T. Weinkauf, N. Teuber, and H. C. Hege, "Vortex and strain skeletons in Eulerian and Lagrangian frames," IEEE Trans. Vis. Comput. Graph. 13(5), 980 (2007).

${ }^{13}$ N. T. Ouellette and J. P. Gollub, "Curvature fields, topology, and dynamics of spatiotemporal chaos," Phys. Rev. Lett. 99, 194502 (2007).

${ }^{14}$ M. Duran-Matute, J. Albagnac, L. P. J. Kamp, and G. J. F. van Heijst, "Dynamics and structure of decaying shallow dipolar vortices," Phys. Fluids 22, 116606 (2010).

${ }^{15}$ U. Bödewadt, "Die Drehströmung über festem Grund," Z. Angew. Math. 20, 241 (1940).

${ }^{16}$ A. Einstein, "Die Ursache der Mäanderbildung der Flußläufe und des sogenannten Baerschen Gesetzes," Naturwiss. 14, $223(1926)$

${ }^{17}$ J. Flòr and G. van Heijst, “An experimental study of dipolar vortex structures in a stratified fluid,” J. Fluid Mech. 279, 101 (1994).

${ }^{18}$ J. M. Nguyen Duc and J. Sommeria, "Experimental characterization of steady two-dimensional vortex couples," J. Fluid Mech. 192, 175 (1988).

${ }^{19}$ D. Sous, N. Bonneton, and J. Sommeria, "Turbulent vortex dipoles in a shallow water layer," Phys. Fluids 16, 2886 (2004).

${ }^{20}$ D. Sous, N. Bonneton, and J. Sommeria, "Transition from deep to shallow water layer; formation of vortex dipoles," Eur. J. Mech. B/Fluids 24, 19 (2005).

${ }^{21}$ P. Billant and J. M. Chomaz, "Experimental evidence for a new instability of a vertical columnar vortex pair in a strongly stratified fluid," J. Fluid Mech. 418, 167 (2000).

${ }^{22}$ V. V. Meleshko and G. J. F. van Heijst, "On Chaplygin's investigations of two-dimensional vortex structures in an inviscid fluid,” J. Fluid Mech. 272, 157 (1994). 